\title{
Intermédialités
}

Histoire et théorie des arts, des lettres et des techniques

Intermediality

History and Theory of the Arts, Literature and Technologies

\section{Transmettre et communiquer}

Chassés-croisés conceptuels à partir de Régis Debray ["Transmettre/ Transmitting", no 5 printemps 2005]

\section{Walter Moser}

Numéro 20, supplément, automne 2012, printemps 2013

traverser

crossing

URI : https://id.erudit.org/iderudit/1023526ar

DOI : https://doi.org/10.7202/1023526ar

Aller au sommaire du numéro

Éditeur(s)

Revue intermédialités (Presses de l’Université de Montréal)

ISSN

1705-8546 (imprimé)

1920-3136 (numérique)

Découvrir la revue

Citer cet article

Moser, W. (2012). Transmettre et communiquer : chassés-croisés conceptuels à partir de Régis Debray ["Transmettre/ Transmitting", no 5 printemps 2005].

Intermédialités / Intermediality, (20), 73-87. https://doi.org/10.7202/1023526ar 


\title{
Transmettre et communiquer \\ Chassés-croisés conceptuels à partir de Régis Debray*
}

\author{
Walter Moser
}

D artons du verbe «transmettre » : un terme, une idée, une notion, un concept.

L'exploration conceptuelle parcourt cette chaîne dans les deux sens: du mot au concept - dans un processus d'abstraction et de clarification qui vise une stabilité univoque - et du concept au mot - dans un processus de ressourcement dans le langage courant et de plongée du concept dans les matériaux langagiers proliférants.

Ce texte propose quelques parcours heuristiques du concept de «transmission » comme nous y convie ce numéro d'Intermédialités. J'ai choisi comme espace d'interrogation, comme lieu de mise à l'essai et comme point de départ concret quelques textes qui relèvent de la médiologie de Régis Debray. Étant donné la haute fréquence du concept de «transmission » dans le réseau multimédiatique des communications de Régis Debray, son élaboration a déjà reçu une grande épaisseur argumentative et sémantique dont nous allons profiter dans les explorations à venir.

\section{I}

Le 9 octobre 2003, Régis Debray donnait une conférence à l'Université d'Ottawa sous le titre «Identité culturelle et mondialisation ». Une lecture préparatoire était recommandée : «Communiquer moins, transmettre plus », qui nous faisait entrer d'office dans le réseau des communications de Debray'. Cette étude au titre programmatique - dont les verbes à l'infinitif devenaient consigne, sinon impératif — semble répondre à une urgence perçue dans la culture contemporaine par

* Les commentaires critiques de Pascal Gin, qui a lu une première version de ce travail, m’ont été d'une grande aide pour la rédaction définitive; je tiens à l'en remercier chaleureusement.

1. Ce texte a été repris dans Régis Debray, "Communiquer moins, transmettre plus », dans Les diagonales du médiologue: transmission, influence, mobilité, Paris, Bibliothèque nationale de France, coll. «Conférences Del Duca», 2001, p. 7-29. 
l'auteur: la communication étant devenue pratique pléthorique, la transmission en souffre et a besoin d'être revalorisée. Pas juste pour rééquilibrer les deux activités, mais, bien plus dramatiquement, en quelque sorte pour sauver l'humanité. Il faut donc communiquer moins et transmettre plus, comme si les deux activités étaient reliées selon une logique de vases communicants: quand l'une monte, l'autre décline.

Aussi Debray caractérise-t-il ces deux activités moyennant une série d'oppositions qui ne dépareilleraient pas la rubrique «antiphrase » dans un dictionnaire des figures rhétoriques. Pour commencer, il nous propose deux définitions minimales: "Communiquer, dans notre jeu de concepts, c'est l'acte de transporter une information dans l'espace, et transmettre, transporter une information dans le temps ${ }^{2}$. »C'est de cette première différence que découle, par la

74 suite, un paradigme dichotomique très élaboré, dont voici quelques entrées :

Communiquer
ubiquité
téléprésence
interactivité
connexion
le contemporain
le droit à l'autonomie

\author{
Transmettre \\ pérennité \\ présence réelle \\ solidarité \\ connivence \\ l'essentiel \\ le devoir d'institution
}

En dramatisant son propos, Debray nous dit ce qui arriverait si on portait chacun des deux côtés du paradigme à son excès :

\author{
les vivants s'éparpillent \\ à tous les vents \\ la culture-bulle \\ le nomade volatile et branché,... \\ surfant sans \\ lest sur le réseau \\ infantilisation par manque d'autorité \\ la désinstitution précarise \\ les êtres humains \\ école-jungle \\ absence de loi
}

\author{
les morts gouvernent \\ les vivants \\ la culture-bunker \\ le paranoïaque cramponné \\ à sa motte de terre, \\ ses tombes et son clan ${ }^{3}$ \\ autorité infantilisante \\ l'institution ossifie \\ les vitalités sociales \\ école-caserne \\ excès de loi
}

2. Régis Debray, «Communiquer moins, transmettre plus», p. 8.

3. Au Québec, on reconnaît ici la figuration antithétique de l'arpenteur et du navigateur, titre d'une publication de Monique LaRue, L'arpenteur et le navigateur, Montréal, Éditions Fides, 1996, qui a été au centre d'un débat public très vif sur la littérature québécoise. 
Dans le diagnostic qu'il fait de la contemporanéité, ce qui apparaît ainsi, du moins rhétoriquement, comme une belle symétrie, serait aujourd'hui déséquilibré par «l'obsession communicante », c'est-à-dire par le peu de poids qu'aurait la transmission face à la communication qui a acquis une importance excessive. Cela s'exprime par un déficit du côté de la transmission :

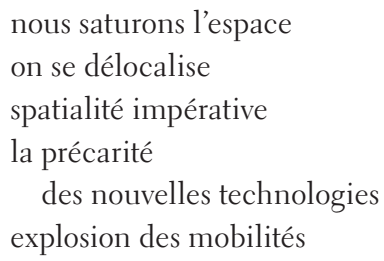

\author{
nous désertons le temps \\ on se déshistoricise \\ historicité évasive \\ la durabilité \\ des anciennes technologies \\ implosion des continuités
}

Bref, il émerge de ce texte un paradigme dichotomique, filé à travers toute son étendue, dont l'effet tranchant est encore rehaussé par les talents stylistiques de Debray, qui a le sens de la formule percutante, voire du slogan.

Résumons cette intervention de Régis Debray dans le débat sur notre société de communication: il constate, du côté du terme de communication, que nous vivons dans une société fragmentée et dispersée en individus autonomes et nomades qui, tout en se servant d'appareils très performants, ont développé des réseaux hypertrophiés de mise en contact, directe ou à distance mais toujours instantanée. Cette société vit, toutefois, dans une temporalité unidimensionnelle faite de la mince couche du temps présent ou, en termes médiatiques, d'un real time sans cesse renouvelé. Par contre, elle occupe maximalement l'espace par la multiplication des contacts communicationnels qui lui procurent une jouissance proche de celle de l'ubiquité. Cette société serait en train d'accumuler un déficit dans la transmission qui consiste essentiellement dans le fait qu'une communauté, occupant un espace déterminé, réussisse à transporter dans le temps les informations essentielles pour la constitution et le maintien de sa propre identité. La priorité d'une telle société résiderait dans la continuité temporelle, garante d'une forte stabilité.

Tant que l'enjeu de ce texte reste dans la construction d'un paradigme dichotomique, on peut, bien sûr, interroger le bien-fondé d'une distinction si tranchée et discuter de sa valeur cognitive, mais la question ne sort guère du cercle étroit d'un débat académique. Toutefois, dans la mesure où Debray s'appuie sur ce paradigme pour avancer un diagnostic sur la société contemporaine et sur ses pratiques culturelles, le fond de la question dépasse le domaine purement épistémique. Notre auteur fait alors figure de prophète du malheur qui dramatise la négativité de notre condition culturelle et nous met en garde contre les tendances lourdes et dangereuses se manifestant dans notre contemporanéité. Debray 
joint ici les rangs de ce que David Thorburn et Henry Jenkins ont appelé récemment «the contemporary doomsayers ${ }^{4}$. Il se range du côté de ceux qui insistent sur la précarité de l'homme contemporain (Ulrich Beck et la société du risques), ou sur la déshistoricisation de la société contemporaine (l'analyse du postmodernisme de Fredric Jameson ${ }^{6}$ ) ou encore sur la culture amnésique qui serait la nôtre (Andreas Huyssens, dans Twilight Memories, parle d'une «culture of amnesia $\left.{ }^{7} »\right)$.

Et pourtant, pour quelqu'un qui craint l'explosion des mobilités attribuées à l'hégémonie du communiquer, et l'implosion concomitante des continuités attribuées à la faiblesse du transmettre, Debray fait figure de communicateur de premier ordre. J'ai parlé plus haut de mon entrée dans son réseau. Son texte fait en effet partie d'une vaste trame d'interventions publiques qui s'échelonnent sur plusieurs années et s'appuient sur une grande variété de supports médiatiques. Il n’est, pour évoquer l'étendue de ce réseau, qu'à considérer quelques-unes des interventions par lesquelles il a communiqué ses idées sur le déséquilibre entre «communiquer» et «transmettre».

Parmi les textes à être présentés en 2003 aux Rencontres de Fès, figure sur le Web sa communication intitulée «La transmission en débat». On y reconnaît le paradigme dichotomique «communiquer vs transmettre », à peu près dans les termes que je viens de présenter, mais en plus concis. Cette fois-ci, le paradigme est, en plus, connecté à la problématique de la mondialisation qui, elle, est dite avoir partie liée avec le côté «communiquer». Communication et mondialisation font cause commune, ce qui donne lieu à une tirade de veine polémique :

Il est dès lors tentant de prendre pour argent comptant la grande illusion de la mondialisation, qui serait la confusion de l'universel et du planétaire, et qu'on pourrait appeler la bévue connective. Parce qu'universel s'oppose à cloison, on voit dans la convergence multimédia et l'interconnexion des P.C. la garantie d'une humanité partout présente à elle-même, unifiée parce que globalisée. Comme si l'interdépendance des neurones dans le système nerveux du village global garantissait l'entente entre globaux-riches et globaux-pauvres. Comme si le maillage pouvait tenir lieu

4. David Thorburn et Henry Jenkins (éds.), Rethinking Media Change: The Aesthetics of Transition, Cambridge, MIT Press, 2003, p. 2. Désormais les références à cet ouvrage seront indiquées par le sigle "RMC" suivi de la page et placées entre parenthèses dans le corps du texte.

5. Ulrich Beck, La société du risque. Sur la voie d'une autre modernité, trad. Laure Bernardi, Paris, Éditions Flammarion-Aubier, coll. «Alto», 2001.

6. Fredric Jameson, Postmodernism: Or the Cultural Logic of Late Capitalism, Durham, Duke University Press, 1991.

7. Andreas Huyssen, Twilight Memories: Marking Time in a Culture of Amnesia, New York, Routledge Press, 1995. 
d'héritage, et de projet. La péréquation des tarifs n'est pas celle des mémoires. Une humanité privée de sa profondeur historique se condamne, se réduit bientôt à la gestion plus ou moins haineuse de ses antagonismes géopolitiques ${ }^{8}$.

La mondialisation permet ici à Régis Debray de donner un nom à l'excès de la communication face au déficit de la transmission. Sa critique débouche alors à nouveau sur l'injonction de «transmettre plus », laissant sous-entendre que la transmission pourrait nous mettre à l'abri de tous les dangers de la mondialisation.

En 2001, c'est d'un autre média dont se servait déjà Régis Debray pour diffuser le même message. Dans un numéro thématique consacré à «Communiquer/ transmettre » des Cahiers de médiologie, on trouve son article «Malaise dans la

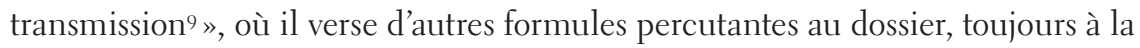
défense de la même cause. Il en résulte une dramatisation additionnelle à ce que nous connaissons déjà de la situation contemporaine:

La formule pourrait être: espaces protégés, durées saccagées. Synchronie gagnée, diachronie perdue. L'espace avale le temps [...]

Molto vivace de la «com», andante des transmissions. Rapidité, Modernité et Communication sont devenus des termes redondants. (MT, p. 18)

Encore une fois la symptomatologie prend la forme d'une dichotomie tranchante, mais dont les deux côtés se sont développés inégalement. La rhétorique de l'antiphrase fait de véritables orgies pour se condenser dans le slogan. Lasymétrie s'accentue, se dramatise: du «malaise dans la transmission» (MT, p. 18) on passe aux «troubles de la transmission» pour aboutir au «déni de la transmission» (MT, p. 21).

En reculant encore de quelques années et en changeant encore une fois de média, on tombe sur le livre Transmettre, de $1997^{10}$. Ce livre représente un aspect du grand projet de médiologie de l'auteur, dont il expose, de toute évidence, une pièce maîtresse. Régis Debray doit y expliquer pourquoi la médiologie ne saurait être réduite ni à l'étude de la communication ou des médias - surtout si on ne

8. Régis Debray, «La transmission en débat», $11^{e}$ Rencontres de Fès, Musée Batha, 7-11 juin 2003, <http://www.fezfestival.org/prg2003/fr/part2003renc/debray.php> (consulté en janvier 2005).

9. Régis Debray, "Malaise dans la transmission", Cahiers de médiologie, $\mathrm{n}^{\circ}{ }^{11}$, "Communiquer / transmettre», novembre 2004, p. 17-33. Désormais les références à cet ouvrage seront indiquées par le sigle «MT» suivi de la page et placées entre parenthèses dans le corps du texte.

10. Régis Debray, Transmettre, Paris, Éditions Odile Jacob, coll. «Le champ médiologique », 1997. 
considère que l'explosion des médias de communication contemporains - ni à l'étude des technologies de communication.

Partout donc le même combat, la même cause défendue et les mêmes erreurs pourfendues. On pourrait encore élargir le réseau en incluant les conférences données à la Bibliothèque nationale de France ${ }^{11}$, les débats à la télévision et jusqu'à l'entrevue qu'il a accordée au journal Wired ${ }^{12}$. Il appert que Régis Debray, pour défendre la cause de la transmission contre la communication, s'avère bien être un redoutable communicateur. Il est présent dans plusieurs médias, il reprend ses idées sous différentes formes, il se déplace dans un vaste réseau communicationnel pour multiplier les lieux où faire entendre son message. Il semble avoir atteint exactement l'état d'ubiquité qu'il questionne en faisant la critique des pratiques de communication permettant d'occuper plusieurs lieux simultanément grâce aux technologies qui nous mettent en contact à distance.

Premier bilan: faites ce que je dis, ne faites pas ce que je fais! ’̀ observer, au fil des années, la croisade de Régis Debray contre le «trop communiquer», en faveur d'un «transmettre plus », on constate un écart entre la théorie et la pratique, entre le diagnostic et le remède, entre l'idéologie de la posture et le pragmatisme de l'exposé. Du moins le voit-on engagé dans une logique identique à celle des anciens antimondialistes devenus altermondialistes, ayant compris soit que le mal qu'ils combattaient les servait bien, soit que leur combat était d'autant plus efficace qu'ils empruntaient les armes de leur ennemi, soit encore, avec un brin de cynisme, que la fin justifiait les moyens.

\section{II}

C'est donc de la pratique même du médiologue que vient le premier avertissement: il se peut que l'opposition entre communiquer et transmettre, si irréconciliable dans les termes, ne soit, en réalité, pas viable dans les faits.

À relire quelques-uns des textes déjà mentionnés, on se rend compte de fait que Régis Debray abonde ici et là en ce sens: la relation entre les deux termes pourrait être de nature moins dichotomique et que, en fait, les belles envolées antithétiques ne réussissent pas vraiment à faire disparaître leurs interconnexions incontournables.

11. Il s'agit de conférences données à la Bibliothèque nationale de France les 4, 6 et 7 décembre 2000, qui ont été reprises dans Régis Débray, Les diagonales du médiologue: transmission, influence, mobilité.

12. Régis Debray, «Revolution in the Revolution. Interview with Andrew Joscelyne », Wired, vol. 3, $\mathrm{n}^{\circ}$ 1, janvier 1995, repris sur le Web: <http://www.wired.com/wired/ archives/3.o1/debray.html>. 
Ainsi trouvons-nous à plus d'une reprise, dans le réseau évoqué, cette même phrase : «Bien sûr, il faut communiquer pour transmettre: condition nécessaire mais non suffisante ${ }^{13}$. » Le constat est rudimentairement succinct et a valeur concessive, certes, mais il n'en établit pas moins une relation d'implication d'un des deux termes dans l'autre. Avec cela, se trouve reconnecté ce que l'opposition des termes voulait couper. La logique dichotomique est entamée, commence à être subvertie.

Mais il y a plus grave. Une des assises conceptuelles de la médiologie de Debray repose sur la distinction entre la matière organisée et l'organisation matérialisée, qu'il développe entre autres dans Transmettre. La matière organisée (M.O.) correspond pour lui à l'appareillage de la culture, dans lequel il range les agencements de communication : ce qui relève du mode sémiotique, du dispositif de diffusion, du support physique, des moyens de transport. Le concept d'organisation matérialisée (O.M.), par contre, nous situe du côté de l'institution; on y trouve les agencements communautaires, «à savoir les diverses formes de cohésion unissant les opérateurs humains d'une transmission ${ }^{14}$ ». C'est cette distinction conceptuelle qui fonde celle entre communication et transmission par laquelle nous sommes entrés dans le réseau.

Or, projetée à ce niveau, la relation entre communiquer et transmettre apparaît sous un jour nouveau, étant donné que les fondements même de cette distinction sont traités selon une logique d'un autre type:

L'interaction M.O./O.M., ou encore la dialectique support/rapports, constitue le point névralgique du schéma proposé. Impossible de traiter séparément l'instance communautaire du dispositif de communication, une sociabilité d'une technicité. Si on osait reconnaître à notre point de vue une originalité et une seule, ce serait ce refus de sacrifier l'un à l'autre l'appareillage et l'institution dans l'analyse des parcours symboliques ${ }^{15}$.

Et, après une digression étymologique sur les deux composantes lexématiques (legere et ligare) dont le mot «religion» serait la confluence sémantique, il conclut par cette question rhétorique: «N'y a-t-il pas implication mutuelle ${ }^{16}$ ? »

Avec cette ouverture sur des relations logiques qui relèvent de l'interaction, de la dialectique et de l'implication mutuelle, nous sommes loin du paradigme dichotomique que j’ai pu extraire du texte «Communiquer moins, transmettre

13. On trouve cette phrase tant dans «Communiquer moins, transmettre plus», p. 8 , que dans «Malaise dans la transmission», p. 17.

14. Régis Debray, Transmettre, p. 30.

15. Régis Debray, Transmettre, p. 41.

16. Régis Debray, Transmettre, p. 41. 
plus ». Si l'on veut maintenir la distinction entre communication et transmission, ces phénomènes d'interaction permettent alors de conserver une véritable dynamique entre les termes, dynamique à laquelle on peut donner plusieurs formes: la présupposition réciproque, l'action réciproque, ou encore la dépendance mutuelle. Il en résulte une relation plus intéressante et plus exigeante à articuler. J'aimerais consacrer le reste de ces réflexions à faire avancer ce travail.

\section{III}

Pour commencer, revenons simplement à nos manières ordinaires de parler. Quels sont, en français, les usages quotidiens du verbe «transmettre » et du substantif «transmission»?

On peut transmettre la vie. Dans un langage biologiquement plus précis, on parle de la transmission d'un gène ou de tout un patrimoine génétique de parents à enfants, d'ancêtres à descendants, mais toujours dans une relation généalogique. Se référant à des systèmes de facture humaine (social, économique, culturel), on dit couramment que les parents transmettent à leurs enfants un nom, une coutume, un héritage ou une succession. Un sujet collectif, tel que «la société », « une classe sociale», « une nation», peut transmettre des valeurs, des normes, d'une génération à une autre, d'une époque à une autre. Avec ces usages des termes, nous restons fidèles à une sémantique du verbe «transmettre » qui est chère à Debray, parce qu'elle a comme axe prioritaire celui du temps. Transmettre apparaît alors comme une opération de passage le long d'un axe temporel qui assure la continuité de ce qui est transmis à travers, et malgré les aléas du temps. Debray parle, métaphoriquement, d'une transmission verticale et, comme nous l'avons déjà vu, il semble vouloir réserver l'usage du verbe pour ce genre de transmission.

Or, la langue française prévoit aussi, outre et par-delà ceux déjà mentionnés, des usages qui s'inscrivent dans une logique différente. C'est le cas quand on transmet non pas un gène mais un virus d'une personne à une autre; quand la transmission devient contagion de corps à corps dans un même espace-temps. Cette transmission-contagion s'accomplit quand il y a coprésence de deux corps, que ces corps soient en contact direct ou connectés par un médium, une matérialité intermédiaire, si ténue soit-elle, comme l'air tant pour les microbes que pour les paroles qui volent. Dans les deux cas, la transmission vient occuper le terrain sémantique que Debray voulait réserver à la communication : quand il y a « coprésence instantanée des contemporains, le "contact" à l'horizontale17 ».

17. Régis Debray, «Communiquer moins, transmettre plus», p. 9. 
Dans le monde inanimé, le médium de ce type de transmission peut être d'une matérialité plus ou moins concrète pour la perception humaine. Ainsi, dans le domaine mécanique, l'arbre de transmission qui permet de transmettre la force du moteur aux roues d'une voiture, doit être d'une matérialité lourde, tandis que, dans les domaines acoustique, optique et électrique, la matérialité des milieux transmetteurs varie beaucoup et a une influence sur la vitesse de la transmission. Dans le domaine des télécommunications, qui s'appuie sur ces transmissions physiques, on transmet des informations d'un point dans l'espace à un autre, d'un destinataire à un destinateur. Avec les progrès technologiques, nos médias de communication se sont dématérialisés : si la poste a encore besoin de transporter beaucoup de papier pour que les messages se transmettent et que la communication puisse avoir lieu, dans les nouveaux médias, cette matérialité se ramène aujourd'hui à des flux électroniques infrasensoriels pour l'être humain qui ne les perçoit que «rematérialisés » sur l'écran pour la perception visuelle et moyennant le haut-parleur pour les perceptions auditives.

Il y a une corrélation entre cette dématérialisation et l'instantanéité, c'està-dire l'unidimensionalité temporelle que Debray attribue à la communication. C'est cette corrélation, entre autres choses, qui permet à Zygmunt Bauman ${ }^{18}$ de distinguer, en s'appuyant sur une métaphore épistémique, une modernité solide d'une modernité liquide: si, dans la première, le temps servait à mesurer la conquête matérielle d'un espace, dans la seconde, grâce aux moyens de communication qui permettent la transmission instantanée d'informations, les relations entre espace et temps se seraient liquéfiées. Il n’y a pratiquement plus de limites à tout ce que les médias contemporains peuvent nous transmettre dans l'instantanéité du real time: un événement sportif, les effets d'une catastrophe naturelle, un discours politique, un message personnel, des manifestations de sympathie ou de colère, un spectacle qui a lieu à l'autre bout du monde, etc.

Le moins qu'on puisse dire c'est que certains usages des termes «transmettre » et «transmission » s'installent de plain-pied dans le champ sémantique que Debray entendait réserver à la communication. Dans son livre Transmettre, nous l'avons déjà vu, il admet le caractère inséparable des deux termes en se référant à leur implication mutuelle. Mais il tient tout de même à privilégier l'un par rapport à l'autre:

[...] si on ne peut, in vivo, séparer complètement les deux notions, on se gardera de les confondre, en subordonnant in vitro la plus moderne à la plus ancienne, qui nous semble à la fois plus intégrative et plus rigoureuse. Un processus de transmission

18. Zygmunt Bauman, Liquid Modernity, Cambridge, Polity Press, 2000. 
inclut nécessairement des faits de communication; l'inverse peut ne pas se produire; le tout primera sur la partie ${ }^{19}$.

Que Debray accorde priorité à la transmission sur la communication, c'est une décision qui découle de son diagnostic de la culture contemporaine, diagnostic auquel je n'adhère pas sans réserves. Ce qui me paraît plus problématique, par contre, c'est qu'il cherche à appuyer cette hiérarchisation des deux termes en faisant valoir la différence de leur structure sémantique : la transmission primerait sur la communication comme le tout sur la partie. Qu'en est-il?

La page titre du numéro 11 des Cahiers de médiologie, en opposant les verbes «communiquer» et «transmettre », fait ressortir l'opposition des deux préfixes séparément, et ce grâce à une disposition typographique particulière :

$\begin{array}{lll}\text { COM } & 1 & \text { TRANS } \\ \text { muniquer } & \backslash & \text { mettre }\end{array}$

Cette graphie a l'avantage de nous rappeler que «transmettre » fait partie d'un paradigme verbal qui a pris beaucoup d'importance, dans les sciences humaines et sociales, eu égard à la description des phénomènes culturels se produisant dans le contexte de la mondialisation. Plus particulièrement, ce paradigme est appelé à capter la grande mobilité qui caractérise aujourd'hui les processus culturels. Il s'agit d'un ensemble de verbes composés du préfixe transet d'un verbe transitif à contenu «phorique » : transmettre, transporter, traduire, transférer, transposer ${ }^{2 \circ}$. Du transport au sens le plus matériel du terme jusqu'aux transferts les plus dématérialisés ${ }^{21}$, ces verbes regroupent et décrivent divers processus qui ont une même structure dynamique : un objet est transporté d'un système à un autre. Une distance est franchie (qu'elle soit de nature temporelle ou spatiale n’apparaît à ce niveau de généralité du paradigme que comme une variation sans pertinence). Les traits pertinents de cette opération sont les suivants:

19. Régis Debray, Transmettre, p. 23.

20. Activement présents au niveau du langage commun, ces verbes font aujourd'hui l'objet d'un travail de (re)conceptualisation. Dans ce numéro spécial, la focalisation est sur «transmettre ». Ailleurs, je participe au travail d'un collectif qui porte sur « transférer» : Pascal Gin, Nicolas Goyer et Walter Moser (éds.), Transfert. Explorations d'un champ conceptuel, Ottawa, Presses de l'Université d'Ottawa, à paraître en 2014.

21. Debray postule un lien de cooccurrence et de corrélation intéressant entre les moyens de locomotion et de médiamotion, lien qui vaudrait la peine d'être exploré plus en profondeur : «[...] les moyens de transport ont un rôle décisif - car la nature d'un régime de locomotion n'est jamais étrangère à l'esprit d'une médiasphère, et au style d'une culture. » (MT, p. 29) 
1. Quelque chose de matériel ou d'immatériel doit être transporté;

2. Ce mouvement de transport doit franchir un seuil, une ligne souvent invisible entre deux instances ou systèmes;

3. Il a lieu dans un médium de transmission qui relève souvent d'un média spécifique;

4. Le mouvement est unidirectionnel.

Or, si la transmission selon Debray s'inscrit parfaitement dans ce paradigme, elle l'active de manière restrictive dans la mesure où la distance à franchir est pensée exclusivement en termes temporels. Il s'agit d'un passage dans le temps entre deux générations, deux époques, deux moments chronologiquement séparés. Cette opération doit justement pouvoir assurer la continuité entre ce qui peut être séparé sur l'axe du temps par la mort ou par l'oubli. Ce trait additionnel postulé par Debray soumet avec force cette transmission de type vertical à la loi de l'irréversibilité et de l'unidirectionalité.

La communication, par contre, est une transmission qui, comme le note Debray, "suppose retour, rencontre, "feedback"22». C'est une transmission dans les deux sens, elle a deux vecteurs par définition: elle va et elle vient. Sans vaet-vient, il n'y a pas de communication, il n’y a que transmission unilatérale de données ou d'informations.

Du coup, la transmission s'avère être la plus faible des deux opérations, celle qui, en tant que transfert d'informations, est contenue deux fois dans l'acte de communication. Si on applique la logique de la partie et du tout à cet état de choses, une inversion s'opère. C'est désormais la communication qui englobe, comme ses parties, deux processus de transmission à vecteurs inversés. Un com- contiendrait au moins deux trans-, sauf que le passage de la transmission d'informations à la communication n'est pas qu'une opération quantitative. Ici, comme ailleurs, s'applique la logique d'un tout qualitatif qui est plus que la somme de ses parties ${ }^{23}$.

À la lumière de ces considérations, la relation entre «transmettre » et «communiquer» ne cesse de se complexifier et de se dynamiser. Si Debray privilégiait

22. Régis Debray, Transmettre, p. 15 .

23. Comme le rappelle, au sujet de la communication, Elena Esposito dans son article «The Arts of Contingency »: «[...] the notion of communication is by now much broader than the mere diffusion of information. » (Elena Esposito, «The Arts of Contingency», vol. 31, $\mathrm{n}^{\circ}$ 1, «Arts of transmission », automne 2004, <http://www.uchicago.edu/ research/jnl-crit-inq/features/artsstatements/arts.esposito.htm>, consulté le 30 janvier 2005) 
la transmission tout en concédant que «bien sûr, il faut communiquer pour transmettre », nous sommes maintenant en état d'amener les deux opérations à un statut d'égalité en affirmant, en retour, que, bien sûr, il faut transmettre pour communiquer. En leur accordant une certaine anthropomorphisation, on est tenté de dire que la relation entre les deux termes, initialement figée dans un binarisme statique, comporte de plus en plus de «communication », c'est-à-dire de « retour, rencontre, "feedback" ».

\section{IV}

Donnant un dernier tour de vis à cette exploration conceptuelle, nous aborderons sa dimension temporelle. C'est de manière emphatique, nous l'avons vu, que

84 Debray situe la temporalité du côté de la transmission, puisque, pour lui, «transmettre c'est transporter une information dans le temps». Encore une fois, nous partons d'une attribution dichotomique très nette:

transmettre: communiquer

temps : espace

Ce schéma, toutefois, est axiologiquement teinté, puisque, implicitement, la temporalité apparaît comme une chose précieuse, un objectif à atteindre: il faut assurer la continuité temporelle. Voilà ce qui fonde également la priorité que Debray accorde à la transmission. Ce qui, évidemment, aura déjà rompu l'équilibre de ce schéma formellement si symétrique. Équilibre qu'il s'agit de rétablir en renforçant les processus qui se déroulent dans le temps, d'où l'injonction «transmettre plus!»

Avec une telle conceptualisation, doublée d'une évaluation négative de la situation culturelle contemporaine et d'un plan d'assainissement, Debray semble prendre parti dans un débat fondamental sur la culture contemporaine. Fredric Jameson en a explicité l'enjeu dans son ouvrage sur la culture postmoderne ${ }^{24}$ : à bien des égards, la culture postmoderne déserte la temporalité, elle est incapable d'assurer la continuité temporelle qui doit être au cour du processus historique et, de ce fait, affaiblit l'historicité de la société contemporaine, au risque de faire advenir une société "privée d'histoire» (bereft of history). Du coup, elle privilégie la spatialité en se manifestant de plus en plus exclusivement dans des relations spatiales. En fait, dit Jameson, c'est l'hyperspace qui est l'expression la plus authentique du postmodernisme. Donc : déficit temporel (hypo-temps) correspond à hypertrophie spatiale. Derrière ce diagnostic apparaît le remède de Debray: «communiquer moins, transmettre plus ».

24. Fredric Jameson, Postmodernism, p. 38-45. 
Mais de quelle temporalité est-il question au juste? Clairement, il y a pour Debray une bonne et une mauvaise temporalité. Il valorise la temporalité du transmettre qui se coule de préférence dans une relation de type généalogique, de descendance et de lignage, où le temps s'organise dans une «verticalité » linéaire et unidirectionnelle qui adopte volontiers la tripartition en passé, présent et futur, tripartition sécularisée et rethéorisée par les théories modernes de l'histoire. Si le passé transmet au présent et que le présent accepte de transmettre à l'avenir - qu'il s'agisse d'informations, de valeurs, d'identités, etc. - , alors la continuité temporelle est assurée. La société humaine reste donc dans le temps.

Du côté du «communiquer », il n'y a pas, en réalité, absence de temps, mais on observe une autre temporalité, évaluée négativement. C'est qu'elle n'est pas généalogique, ni linéaire, ni orientée. Elle est faite de discontinuités, de minceur, mais aussi d'intensités et de vitesses. Sa dimension dominante est l'instant présent. Encore une fois, Debray adhère ici à une manière doxique de penser: n'est reconnu comme temporalité qu'un certain ordre temporel. Dès que cet ordre est dérangé, ou qu'on en sort, on parle alors soit, radicalement, d'une absence du temps et d'une perte de l'histoire, soit, plus axiologiquement, d'une temporalité amoindrie et par conséquent négative.

Certes, je l'ai argumenté ailleurs' ${ }^{25}$, les troubles temporels que nous relevons dans la contemporanéité peuvent être vus comme l'affaiblissement d'une temporalité moderne. À cet égard, je partage en partie la symptomatologie de certains désordres temporels que dresse Debray. Mais ces désordres qui ont pour nom accélération, anachronisme, présentéité, instantanéité, soudaineté, entre autres, sont autant de motifs positifs pour penser le temps autrement, pour concevoir des alternatives dans l'ordre temporel. Entre autres choses, on découvre en fait que la minceur du temps présent contient une épaisseur qualitative et une complexité structurale à peine soupçonnées ${ }^{26}$; que l'anachronisme, vice méthodologique de l'historiographie, renvoie aussi à une anachronie qui pourrait être ontologiquement plus vraie que la linéarité progressive.

Je serai donc bien plus prudent que Debray pour ce qui est d'interpréter les particularités temporelles observables dans nos sociétés de communication comme des absences ou comme des négativités temporelles. Et même si l'on

25. Walter Moser, "Time is out of Joint": Temporal Disorders in the Late Modern Condition ", dans Djelal Kadir, Dorothea Löbbermann (dirs.), Other Modernisms in an Age of Globalization, Heidelberg, Universitätsverlag Carl Winter, 2002, p. 183-207.

26. C'est cette épaisseur et cette complexité que tentent de capter conceptuellement des termes comme la «non-contemporanéité» de Ernst Bloch, «l'hybridité temporelle» de Néstor García Canclini et jusqu’à la Jetztzeit de Walter Benjamin. 
adopte l'argumentation de Debray, il n'est pas sûr que ce côté de sa dichotomie soit si dépourvu de continuités temporelles. Considérons à ce sujet les médias qui sont en premier lieu pour Debray des outils de diffusion de l'information. En tant que moyens de diffusion massive et de télécommunication (on pense au téléphone, à la radio, à la télévision, à la presse écrite, au cinéma, à l'Internet), ils s'inscrivent dans le paradigme hégémonique de la communication que Debray a repéré dans la société contemporaine. De ce fait, ils seraient à la fois les opérateurs et les représentants des discontinuités temporelles qui sont une des caractéristiques intrinsèques de ce paradigme.

En guise de conclusion, j'aimerais développer ces réflexions, produites jusqu' ici à partir des travaux de Régis Debray, en recourant à de récentes analyses qui problématisent le fonctionnement et l'histoire des médias, dans la mesure où elles nous montrent plus de continuités temporelles que Debray n’est disposé à en reconnaître du côté du terme "communiquer». Je prends à titre d'exemple le livre sur les changements médiatiques Rethinking Media Change : The Aesthetics of Transition. Déjà le mot du titre, «transition ", annonce l'intervention particulière de ce livre dans l'histoire des médias: les auteurs n'adhèrent pas à la thèse des changements abrupts et des émergences subites; voilà pourquoi ils proposent de repenser le processus historique des changements médiatiques:

Challenging the assumption that new technologies displace older systems with decisive suddenness and have a revolutionary impact on society, the essays in this book see media change as an accretive, gradual process, always a mix of tradition and innovation, in which emerging and established systems interact, shift, and collude with one another. (RMC, p. IX-X)

Plus loin, dans l'introduction, ils précisent cette prise de position programmatique:

If emerging media are often experimental and self-reflexive, they are also inevitably and centrally imitative, rooted in the past, in the practices, formats and deep assumptions of their predecessors. (RMC, p. 7)

Avec cette nouvelle insistance sur le "principe de la continuité » (RMC, p. 7 et 12), c'est la continuité temporelle attribuée en propre par Debray à l'autre côté de sa dichotomie que Thorburn et Jenkins inscrivent dans l'existence et l'émergence même de l'appareillage médiatique qui sous-tend et soutient les processus de communication. La differentia specifica, et surtout l'apanage positif du «transmettre », se retrouve donc de la sorte fermement installée dans la demeure conceptuelle du terme opposé: les médias évoluent et se transforment selon une logique propre qui inclut la transmission d'un héritage ainsi que sa transformation le long d'un axe temporel. Ainsi s'avère-t-il difficile, si on prend au sérieux 
cette historicité des médias de communication et de télécommunication, de localiser la communication sur un continent sans histoire.

Il y a plus : cette continuité temporelle vient même se loger dans le fonctionnement, dans l'autoreprésentation des médias et dans les discours qui circulent sur eux. C'est ce à quoi nous a rendus attentifs le livre récent de Jay Bolter et Richard Grusin intitulé Remediation ${ }^{27}$. Le terme «remédiation » est redoutablement polysémique. Ses faisceaux de signification les plus pertinents pour notre propos affirment que tout nouveau média s'installe sur la base de ses prédécesseurs, les imite et prétend en améliorer le fonctionnement; il en reproduit les fonctions en les transposant sur de nouveaux supports matériels et techniques. À la limite, pour se faire accepter, il se fait passer pour son prédécesseur. Le média dispose donc d'un espace de mémoire interne qu'il peut activer et occuper pour des fins d'implantation et d'autoreprésentation. Il se donne ainsi une continuité dans le temps qui confirme, de l'intérieur, celle que l'historien des médias semble lui découvrir sous son regard extérieur. Et cette continuité du média, soit dit en passant, est de nature constitutivement intermédiale, puisqu'elle met en interaction divers dispositifs médiatiques le long d'un axe temporel.

C'est ainsi qu'un ordinateur dont le fonctionnement technique est basé sur la numérisation électronique se fait passer pour un power book en référence à une génération antérieure d'appareillages médiatiques. C'est ainsi aussi que Wim Wenders, dans son film Aufzeichnungen zu Kleidern und Städten (A Notebook on Cities and Clothes, 1989), peut mettre le bloc-notes du couturier en parallèle et à égalité fonctionnelle avec l'appareil-vidéo du cinéaste: la vidéo numérique rend le même service (au cinéaste) que le bloc-notes (au couturier) dans le processus de création. Deux technologies et pratiques d'annotation historiquement décalées, mais intervenant dans le même espace-temps d'un film, se trouvent ainsi téléscopées dans une remédiation complexe. Bolter et Grusin vont jusqu’à établir un lien de remédiation entre le windowed screen du poste de télévision CNN et la fenêtre dans la peinture, et plus particulièrement dans le portrait, de la Renaissance... Quoi qu'il en soit, en nous sensibilisant aux processus de remédiation, ce livre, à l'inverse des travaux de Debray, nous a fait découvrir une continuité temporelle interne aux médias qui inscrivent ainsi, dans leur fonctionnement, leur propre histoire, même si leur performance technique renforce, par ailleurs, la dimension temporelle de l'instantanéité dans la culture contemporaine.

27. Jay Bolter, Richard Grusin, Remediation: Understanding New Media, Cambridge, MIT Press, 1999. 